\title{
Research Article \\ Properties of Recurrent Equations for the Full-Availability Group with BPP Traffic
}

\author{
Mariusz Głąbowski, Maciej Stasiak, and Joanna Weissenberg \\ Communication and Computer Networks, Faculty of Electronics and Telecommunications, \\ Poznan University of Technology, ul. Polanka 3, 60-965 Poznan, Poland \\ Correspondence should be addressed to Mariusz Głąbowski, mariusz.glabowski@put.poznan.pl \\ Received 27 April 2011; Accepted 1 August 2011 \\ Academic Editor: Yun-Gang Liu
}

Copyright (C) 2012 Mariusz Głąbowski et al. This is an open access article distributed under the Creative Commons Attribution License, which permits unrestricted use, distribution, and reproduction in any medium, provided the original work is properly cited.

The paper proposes a formal derivation of recurrent equations describing the occupancy distribution in the full-availability group with multirate Binomial-Poisson-Pascal (BPP) traffic. The paper presents an effective algorithm for determining the occupancy distribution on the basis of derived recurrent equations and for the determination of the blocking probability as well as the loss probability of calls of particular classes of traffic offered to the system. A proof of the convergence of the iterative process of estimating the average number of busy traffic sources of particular classes is also given in the paper.

\section{Introduction}

Dimensioning and optimization of integrated networks, that is, Integrated Services Digital Networks (ISDN) and Broadband ISDN (B-ISDN) as well as wireless multiservice networks (e.g., UMTS), have recently developed an interest in multirate models [1-5]. These models are discrete models in which it is assumed that the resources required by calls of particular traffic classes are expressed as the multiple of the so-called Basic Bandwidth Units (BBUs). The BBU is defined as the greatest common divisor of the resources demanded by all call streams offered to the system $[6,7]$.

Multirate systems can be analysed on the basis of statistical equilibrium equations resulting from the multidimensional Markov process that describe the service process in the considered systems [8-13]. Such an approach, however, is not effective because of the quickly increasing-along with the system's capacity—number of states in which a multidimensional Markov process occurring within the system can take place [14]. Consequently, for an analysis of multirate systems, there are used methods based on the convolution algorithm 
$[11,15]$ and the recurrent methods in which the multidimensional service process-occurring in the considered systems-is approximated by one-dimensional Markov chain [16-21]. The convolution methods allow us to determine exactly the occupancy distribution in the so-called full-availability systems servicing traffic streams with arbitrary distributions (i.e., systems with state-independent admission process and with both state-independent and state-dependent arrival processes). In the case of the systems with state-dependent admission process (i.e., the system in which the admission of a new call is conditioned not only by the sufficient number of free BBUs but also by the structure of the system and the introduced admission policy) the convolution methods lead to elaboration of approximate methods with quite high computational complexity [22, 23].

Nowadays, in the analysis and optimization of multirate systems, the recurrent algorithms are usually used. This group of algorithms is based on the approximation of the multidimensional service process in the considered system by the one-dimensional Markov chain. Such approach leads to a determination of the occupancy distribution in systems with state-independent admission process and state-independent arrival process (in teletraffic engineering such system is called the full-availability group with Erlang traffic streams) on the basis of simple Kaufman-Roberts recurrence [24, 25] and its modifications [16-19, 26, 27]. One of them, the so-called Delbrouck recurrence [18], allows us to determine the occupancy distribution in the system with state-independent admission process (the full-availability group) and BPP traffic streams. The research on the full-availability group model, started by Delbrouck, was subsequently continued, for example, in [12, 28-30].

Because of the simplicity of the Kaufman-Roberts equation, in many works the attempts of its modification in order to analyse the systems with BPP traffic were undertaken. In [13] the modified form of the Kaufman-Roberts equation that makes the value of offered traffic dependent on the number of active sources was presented. In [31] the approximation of the number of active sources with their mean values in relation to the total value of occupied resources in particular states of the system was proposed. In [32], on the basis of the method proposed in [31], the Kaufman-Roberts equation was generalized for systems with BPP traffic and state-dependent call admission process. The accuracy of the method for modelling systems with multirate BPP traffic-further on called the Multiple Iteration Method-BPP (MIM-BPP)—proposed in [32] was verified in simulations for systems with both state-independent and state-dependent call admission process. In publications issued so far, no attempt to formally prove the correctness of the MIM-BPP assumptions was taken up.

The aim of this paper is to formally prove that the MIM-BPP algorithm [32], considered earlier as an approximate algorithm, is exact. To this purpose we derive recurrent equations describing the occupancy distribution in the full-availability group with multirate BPP traffic. We are going to demonstrate at the same time that the number of calls of particular Engset and Pascal classes appearing in equations that determine the occupancy distribution is exactly determined with their average values. Additionally, we intend to prove the convergence of the iterative process of estimating the average number of busy traffic sources of particular classes.

The paper is organized as follows. Section 2 presents an analysis of the call admission and the call arrival process in the full-availability group with BPP traffic at the micro- and macrostate level. In Section 3 an iterative method for estimating the average number of busy traffic sources of particular classes is presented, and its convergence is proved. The paper ends with a summary contained in Section 4. 


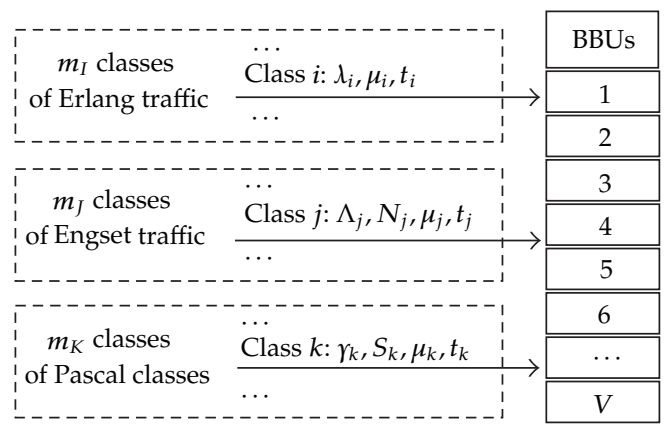

Figure 1: Full-availability group with the Erlang, Engset, and Pascal traffic stream.

\section{Full-Availability Group with BPP Traffic}

\subsection{Basic Assumptions}

Let us consider a model of the full-availability group with the capacity of $V$ BBUs (Figure 1). The group is offered traffic streams of three types: $m_{I}$ Erlang streams (Poisson distribution of call streams) from the set $I=\left\{1, \ldots, i, \ldots, m_{I}\right\}, m_{J}$ Engset streams (binomial distribution of call streams) from the set $J=\left\{1, \ldots, j, \ldots, m_{J}\right\}$, and $m_{K}$ Pascal streams (negative binomial distribution of call stream) from the set $K=\left\{1, \ldots, k, \ldots, m_{K}\right\}$. In the paper it has been adopted that the letter " $i$ " denotes any class of Erlang traffic, letter " $j$ " any class of Engset traffic, and letter " $k$ " any class of Pascal traffic, whereas the letter " $c$ " any traffic class. (In relation to the ITU-T recommendations [11], all types of discussed traffic are defined collectively by the term BPP traffic. Thus, we use the term BPP when we talk about all traffic types cumulatively, whereas when we consider single traffic streams, then, because our study is focused on systems with limited capacity only, we use the terms Erlang, Engset, and Pascal streams.) The number of BBUs demanded by calls of class $c$ is denoted by $t_{c}$.

The call arrival rate for Erlang traffic of class $i$ is equal to $\lambda_{i}$. The parameter $\lambda_{j}\left(y_{j}\right)$ determines the call intensity for the Engset traffic stream of class $j$, whereas the parameter $\lambda_{k}\left(z_{k}\right)$ determines the call intensity for Pascal traffic stream of class $k$. The arrival rates $\lambda_{j}\left(y_{j}\right)$ and $\lambda_{k}\left(z_{k}\right)$ depend on the number of $y_{j}$ and $z_{k}$ of currently serviced calls of class $j$ and $k$. In the case for Engset stream, the arrival rate of class $j$ stream decreases with the number of serviced traffic sources:

$$
\lambda_{j}\left(y_{j}\right)=\left(N_{j}-y_{j}\right) \Lambda_{j}
$$

where $N_{j}$ is the number of Engset traffic sources of class $j$, while $\Lambda_{j}$ is the arrival rate of calls generated by a single free source of class $j$. In the case of Pascal stream of class $k$, the arrival rate increases with the number of serviced sources:

$$
\lambda_{k}\left(z_{k}\right)=\left(S_{k}+z_{k}\right) \gamma_{k}
$$

where $S_{k}$ is the number of Pascal traffic sources of classes $k$, while $\gamma_{k}$ is the arrival rate of calls generated by a single free source of class $k$. 


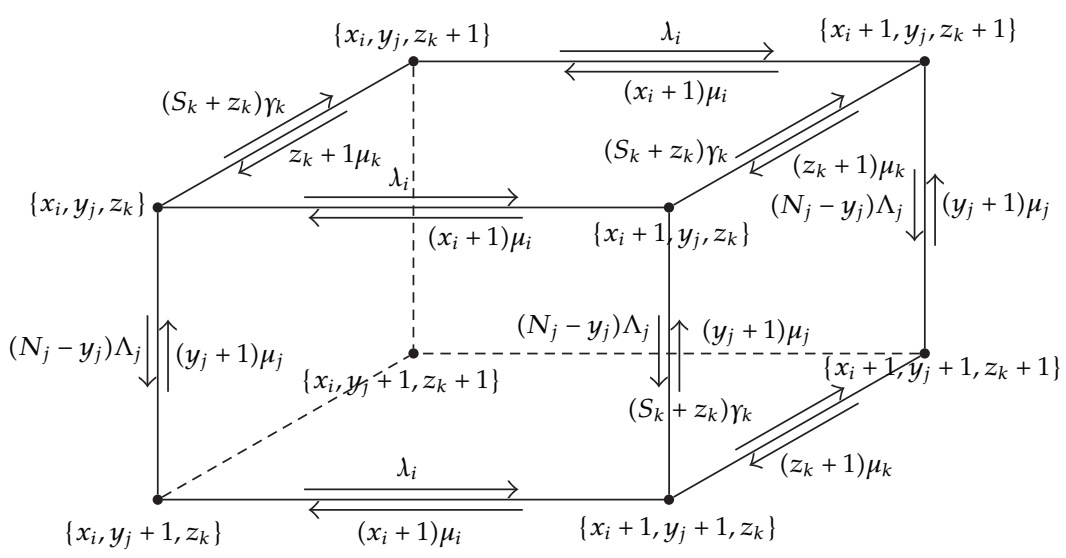

Figure 2: Fragment of a diagram of Markov process in the full-availability group with BPP traffic.

The total intensity of Erlang traffic of class $i$ offered to the group amounts to

$$
A_{i}=\frac{\lambda_{i}}{\mu_{i}}
$$

whereas the intensity of Engset traffic $\alpha_{j}$ and Pascal traffic $\beta_{k}$ of class $j$ and $k$, respectively, offered by one free source, is equal to

$$
\alpha_{j}=\frac{\Lambda_{j}}{\mu_{j}}, \quad \beta_{k}=\frac{r_{k}}{\mu_{k}} .
$$

In (2.3) and (2.4) the parameter $\mu$ is the average service intensity with the exponential distribution.

\subsection{The Multidimensional Erlang-Engset-Pascal Model at the Microstate Level}

Let us consider now a fragment of the multidimensional Markov process in the fullavailability group with the capacity of $V$ BBUs presented in Figure 2. The group is offered traffic streams of three types: Erlang, Engset, and Pascal. Each microstate of the process $\left\{x_{1}, \ldots, x_{i}, \ldots, x_{m_{I}}, y_{1}, \ldots, y_{j}, \ldots, y_{m_{I}}, z_{1}, \ldots, z_{k}, \ldots, z_{m_{K}}\right\}$ is defined by the number of serviced calls of each of the classes of offered traffic, where $x_{i}$ denotes the number of serviced calls of the Poisson stream of class $i$ (Erlang traffic), $y_{j}$ denotes the number of serviced calls of the binomial stream of class $j$ (Engset traffic), whereas $z_{k}$ determines the number of serviced calls of the negative binomial stream of class $k$ (Pascal traffic). To simplify the description, the microstate probability will be denoted by the symbol $p\left(x_{i}, y_{j}, z_{k}\right)$.

The multidimensional service process in the Erlang-Engset-Pascal model is a reversible process. In concordance with Kolmogorov reversibility test considering any cycle for the microstates shown in Figure 2, we always obtain equality in the intensity of transitions (streams) in both directions. The property of reversibility implies the local equilibrium 
equations between any of the two neighbouring states of the process. Such equations for the Erlang stream of class $i$, the Engset stream of class $j$, and Pascal stream of class $k$ can be written in the following way (Figure 2):

$$
\begin{gathered}
x_{i} \mu_{i} p\left(x_{i}, y_{j}, z_{k}\right)=\lambda_{i} p\left(x_{i}-1, y_{j}, z_{k}\right), \\
y_{j} \mu_{j} p\left(x_{i}, y_{j}, z_{k}\right)=\left[N_{j}-\left(y_{j}-1\right)\right] \Lambda_{j} p\left(x_{i}, y_{j}-1, z_{k}\right), \\
z_{k} \mu_{k} p\left(x_{i}, y_{j}, z_{k}\right)=\left[S_{k}+\left(z_{k}-1\right)\right] \gamma_{k} p\left(x_{i}, y_{j}, z_{k}-1\right) .
\end{gathered}
$$

Since the call streams offered to the group are independent, we can add up, for the microstate $\left\{x_{i}, y_{j}, z_{k}\right\}$, all $m_{I}$ equations of type (2.5) for the Erlang streams, $m_{J}$ equations of type (2.6) for the Engset streams, and $m_{K}$ equations of type (2.7) for the Pascal streams. Additionally, taking into consideration traffic intensity (see (2.3) and (2.4)), we get

$$
\begin{aligned}
& p\left(x_{i}, y_{j}, z_{k}\right)\left[\sum_{i=1}^{m_{I}} x_{i} t_{i}+\sum_{j=1}^{m_{J}} y_{j} t_{j}+\sum_{k=1}^{m_{K}} z_{k} t_{k}\right] \\
& =\sum_{i=1}^{m_{I}} A_{i} t_{i} p\left(x_{i}-1, y_{j}, z_{k}\right)+\sum_{j=1}^{m_{J}}\left[N_{j}-\left(y_{j}-1\right)\right] \alpha_{j} t_{j} p\left(x_{i}, y_{j}-1, z_{k}\right) \\
& \quad+\sum_{k=1}^{m_{K}}\left[S_{k}+\left(z_{k}-1\right)\right] \beta_{k} t_{k} p\left(x_{i}, y_{j}, z_{k}-1\right) .
\end{aligned}
$$

\subsection{The Full-Availability Group with BPP Traffic at the Macrostate Level}

It is convenient to consider the multidimensional process occurring in the considered system at the level of the so-called macrostates. Each macrostate $n$ determines the number of $n$ busy BBUs in the considered group, regardless of the number of serviced calls of particular classes. Therefore, each of the microstates $\left\{x_{i}-1, y_{j}, z_{k} \cdots\right\}$ is associated with such a macrostate in which the number of busy BBUs is decreased by $t_{i}$ BBUs, necessary to set up a connection of class $i$, that is, with such a macrostate in which the number of busy BBUs equals $n-t_{i}$. The following equation is then fulfilled:

$$
\sum_{\substack{c=1 \\ c \neq i}}^{m} x_{c} t_{c}+\left(x_{i}-1\right) t_{i}=\sum_{c=1}^{m} x_{c} t_{c}-t_{i}=\left(n-t_{i}\right),
$$

where $m$ determines the number of all traffic classes offered to the system, that is, $m=m_{I}+$ $m_{J}+m_{K}$.

The macrostate probability $P(n)$ defines then the occupancy probability of $n$ BBUs of the group and can be expressed as the aggregation of the probabilities of appropriate microstates:

$$
P(n)=\sum_{\Omega(n)} p\left(x_{i}, y_{j}, z_{k}\right)
$$


where $\Omega(n)$ is a set of all such subsets $\left\{x_{i}, y_{j}, z_{k}\right\}$ that fulfil the following equation:

$$
n=\sum_{i=1}^{m_{I}} x_{i} t_{i}+\sum_{j=1}^{m_{J}} y_{j} t_{j}+\sum_{k=1}^{m_{K}} z_{k} t_{k} .
$$

The definition of the macrostate (2.11) makes it possible to convert (2.8) into the following form:

$$
\begin{aligned}
n p\left(x_{i}, y_{j}, z_{k}\right)= & \sum_{i=1}^{m_{I}} A_{i} t_{i} p\left(x_{i}-1, y_{j}, z_{k}\right) \\
& +\sum_{j=1}^{m_{J}}\left[N_{j}-\left(y_{j}-1\right)\right] \alpha_{j} t_{j} p\left(x_{i}, y_{j}-1, z_{k}\right) \\
& +\sum_{k=1}^{m_{K}}\left[S_{k}+\left(z_{k}-1\right)\right] \beta_{k} t_{k} p\left(x_{i}, y_{j}, z_{k}-1\right) .
\end{aligned}
$$

Adding on both sides all microstates that belong to the set $\Omega(n)$, we get

$$
\begin{aligned}
n \sum_{\Omega(n)} p\left(x_{i}, y_{j}, z_{k}\right)= & \sum_{i=1}^{m_{I}} A_{i} t_{i} \sum_{\Omega(n)} p\left(x_{i}-1, y_{j}, z_{k}\right) \\
& +\sum_{j=1}^{m_{J}}\left[N_{j}-\left(y_{j}-1\right)\right] \alpha_{j} t_{j} \sum_{\Omega(n)} p\left(x_{i}, y_{j}-1, z_{k}\right) \\
& +\sum_{k=1}^{m_{K}}\left[S_{k}+\left(z_{k}-1\right)\right] \beta_{k} t_{k} \sum_{\Omega(n)} p\left(x_{i}, y_{j}, z_{k}-1\right) .
\end{aligned}
$$

Following the application of the definition of macrostate probability, expressed by (2.10), we are in a position to convert (2.13) as follows:

$$
\begin{aligned}
n P(n)= & \sum_{i=1}^{m_{I}} A_{i} t_{i} P\left(n-t_{i}\right)+\sum_{j=1}^{m_{J}}\left[N_{j}-\left(y_{j}-1\right)\right] \alpha_{j} t_{j} \\
& \times \sum_{\Omega(n)} p\left(x_{i}, y_{j}-1, z_{k}\right)+\sum_{k=1}^{m_{K}}\left[S_{k}+\left(z_{k}-1\right)\right] \beta_{k} t_{k} \\
& \times \sum_{\Omega(n)} p\left(x_{i}, y_{j}, z_{k}-1\right)
\end{aligned}
$$


Mathematical Problems in Engineering

$$
\begin{aligned}
= & \sum_{i=1}^{m_{I}} A_{i} t_{i} P\left(n-t_{i}\right)+\sum_{j=1}^{m_{J}} \alpha_{j} t_{j} \sum_{\Omega(n)}\left[N_{j}-\left(y_{j}-1\right)\right] \frac{p\left(x_{i}, y_{j}-1, z_{k}\right)}{\sum_{\Omega(n)} p\left(x_{i}, y_{j}-1, z_{k}\right)} \\
& \times \sum_{\Omega(n)} p\left(x_{i}, y_{j}-1, z_{k}\right)+\sum_{k=1}^{m_{K}} \beta_{k} t_{k} \sum_{\Omega(n)}\left[S_{k}+\left(z_{k}-1\right)\right] \\
& \times \frac{p\left(x_{i}, y_{j}, z_{k}-1\right)}{\sum_{\Omega(n)} p\left(x_{i}, y_{j}, z_{k}-1\right)} \sum_{\Omega(n)} p\left(x_{i}, y_{j}, z_{k}-1\right),
\end{aligned}
$$

where $P\left(n-t_{c}\right)=0$, if $n<t_{c}$, and the value $P(0)$ ensues from the normative condition $\sum_{n=0}^{V} P(n)=1$.

In (2.14) the sums

$$
\begin{aligned}
& \sum_{\Omega(n)}\left[y_{j}-1\right] \frac{p\left(x_{i}, y_{j}-1, z_{k}\right)}{\sum_{\Omega(n)} p\left(x_{i}, y_{j}-1, z_{k}\right)}=\overline{y_{j}-1} \\
& \sum_{\Omega(n)}\left[z_{k}-1\right] \frac{p\left(x_{i}, y_{j}, z_{k}-1\right)}{\sum_{\Omega(n)} p\left(x_{i}, y_{j}, z_{k}-1\right)}=\overline{z_{k}-1}
\end{aligned}
$$

determine the value of the average number $\overline{y_{j}-1}, \overline{z_{k}-1}$ of calls of class $j$ and $k$ in occupancy states (macrostates) $n-t_{j}$ and $n-t_{k}$, respectively. In order to determine the relationship between the number of serviced calls of particular traffic classes and the macrostate (for which the average values $\overline{y_{j}-1}$ and $\overline{z_{k}-1}$ are determined), in the subsequent part of the paper we have adopted the following notations:

$$
\begin{aligned}
& \overline{y_{j}-1}=r_{j}\left(n-t_{j}\right), \\
& \overline{z_{k}-1}=r_{k}\left(n-t_{k}\right) .
\end{aligned}
$$

Taking into consideration (2.15) and (2.16), we can rewrite (2.14) in the following way:

$$
\begin{aligned}
n P(n)= & \sum_{i=1}^{m_{I}} A_{i} t_{i} P\left(n-t_{i}\right)+\sum_{j=1}^{m_{J}} \alpha_{j} t_{j}\left[N_{j}-\left(\overline{y_{j}-1}\right)\right] P\left(n-t_{j}\right) \\
& +\sum_{k=1}^{m_{K}} \beta_{k} t_{k}\left[S_{k}+\left(\overline{z_{k}-1}\right)\right] P\left(n-t_{k}\right) \\
= & \sum_{i=1}^{m_{I}} A_{i} t_{i} P\left(n-t_{i}\right)+\sum_{j=1}^{m_{J}} \alpha_{j} t_{j}\left[N_{j}-r_{j}\left(n-t_{j}\right)\right] P\left(n-t_{j}\right) \\
& +\sum_{k=1}^{m_{K}} \beta_{k} t_{k}\left[S_{k}+r_{k}\left(n-t_{k}\right)\right] P\left(n-t_{k}\right) .
\end{aligned}
$$




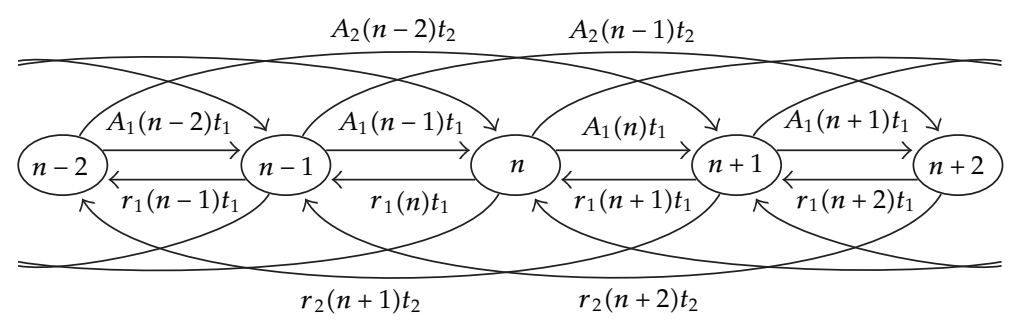

Figure 3: A fragment of the one-dimensional Markov process in the full-availability group with multirate traffic, servicing two call streams $\left(m=1, t_{1}=1 \mathrm{BBU}, t_{2}=3 \mathrm{BBUs}\right)$.

In (2.18) the value of Engset traffic of class $j$ and Pascal traffic of class $k$ depends on the occupancy state of the system. Let us introduce the following notation for the offered traffic intensity in appropriate occupancy states of the group:

$$
\begin{gathered}
A_{i}(n)=A_{i}, \\
A_{j}(n)=\alpha_{j}\left[N_{j}-\left(r_{j}(n)\right)\right], \\
A_{k}(n)=\beta_{k}\left[S_{k}+\left(r_{k}(n)\right)\right] .
\end{gathered}
$$

Formula (2.18) can be now finally rewritten to the following form:

$$
\begin{aligned}
n P(n)= & \sum_{i=1}^{m_{I}} A_{i}\left(n-t_{i}\right) t_{i} P\left(n-t_{i}\right)+\sum_{j=1}^{m_{J}} A_{j}\left(n-t_{j}\right) t_{j} P\left(n-t_{j}\right) \\
& +\sum_{k=1}^{m_{K}} A_{k}\left(n-t_{k}\right) t_{k} P\left(n-t_{k}\right) \\
= & \sum_{c=1}^{m} A_{c}\left(n-t_{c}\right) t_{c} P\left(n-t_{c}\right) .
\end{aligned}
$$

\section{Modelling the Full-Availability Group}

\subsection{Average Number of Serviced Calls of Class $c$ in State $n$}

In order to determine the average number of calls serviced in particular states of the system, let us consider a fragment of the one-dimensional Markov chain presented in Figure 3 and corresponding to the recurrent determination of the occupancy distribution in the fullavailability group on the basis of (2.22). The diagram presented in Figure 3 shows the service process in the group with two call streams ( $\left.m=2, t_{1}=1 \mathrm{BBU}, t_{2}=3 \mathrm{BBUs}\right)$.

Let us notice that each state of the Markov process in the full-availability group (Figure 3) fulfils the following equilibrium equation:

$$
P(n)\left[\sum_{c=1}^{m} A_{c}(n) t_{c}+\sum_{c=1}^{m} t_{c} r_{c}(n)\right]=\sum_{c=1}^{m} A_{c}\left(n-t_{c}\right) t_{c} P\left(n-t_{c}\right)+\sum_{c=1}^{m} t_{c} r_{c}\left(n+t_{c}\right) P\left(n+t_{c}\right)
$$


where $r_{c}(n)$ is the average number of calls of a given class being serviced in state $n$. From (3.1) it results that the sum of all service streams outgoing from state $n$ towards lower states is equal to $n$ :

$$
n=\sum_{c=1}^{m} t_{c} r_{c}(n)
$$

On the basis of (2.22) and (3.2), Formula (3.1) can be rewritten in the following form:

$$
\sum_{c=1}^{m} A_{c}(n) t_{c} P(n)=\sum_{c=1}^{m} t_{c} r_{c}\left(n+t_{c}\right) P\left(n+t_{c}\right)
$$

Equation (3.3) is a balance equation between the total stream of calls outgoing from state $n$ and the total service stream coming in to state $n$. This equation is fulfilled only when the local equilibrium equations for streams of particular traffic classes are fulfilled:

$$
A_{c}(n) t_{c} P(n)=t_{c} r_{c}\left(n+t_{c}\right) P\left(n+t_{c}\right) .
$$

On the basis of (3.4), the average number of calls of class $c$ in state $n+t_{c}$ of the group may be finally expressed in the following way:

$$
r_{c}\left(n+t_{c}\right)= \begin{cases}A_{c}(n) \frac{P(n)}{P\left(n+t_{c}\right)}, & \text { for } n+t_{c} \leq V, \\ 0, & \text { for } n+t_{c}>V .\end{cases}
$$

\subsection{MIM-BPP Method}

Let us notice that, in order to determine the parameter $r_{c}(n)$, it is necessary to determine first the occupancy distribution $P(n)$. Simultaneously, in order to determine the occupancy distribution $P(n)$, it is also necessary to determine the value $r_{c}(n)$. This means that $(2.22)$ and (3.5) form a set of confounding equations that can be solved with the help of iterative methods [32]. Let $P^{(l)}(n)$ denote the occupancy distribution determined in step $l$, and let $r_{c}^{(l)}(n)$ denote the average number of serviced calls of class $c$, determined in step $l$. In order to determine the initial value of the parameter $r_{c}^{(0)}(n)$, it is assumed, according to [32], that the traffic intensities of Engset and Pascal classes do not depend on the state of the system and are equal to the traffic intensity offered by all free Engset sources of class $j$ and Pascal sources of class $k$, respectively: $A_{j}^{(0)}(n)=A_{j}=N_{j} \alpha_{j}, A_{k}^{(0)}(n)=A_{k}=S_{k} \beta_{k}$. When we have the initial values of offered traffic, in the subsequent steps, we are in a position to determine the occupancy distribution, taking into account the dependence of the arrival process on the state of the system. The iteration process finishes when the assumed accuracy is obtained.

On the basis of the reasoning presented above, in [32] the MIM-BPP method for a determination of the occupancy distribution, blocking probability, and the loss probability in the full-availability group with BPP traffic is proposed. The MIM-BPP method can be presented in the form of the following algorithm. 
Algorithm 3.1 (MIM-BPP method). Consider the following steps.

(1) Determination of the value of Erlang traffic $A_{i}$ of class $i$ on the basis of (2.3).

(2) Setting the iteration step: $l=0$.

(3) Determination of initial values of the number $r_{j}^{(l)}(n)$ of Engset serviced calls of class $j$ and the number $r_{k}^{(l)}(n)$ of Pascal serviced calls of class $k$ :

$$
\forall_{0 \leq n \leq V} \quad\left(\forall_{1 \leq j \leq m_{J}} r_{j}^{(l)}(n)=0, \forall_{1 \leq k \leq m_{K}} r_{k}^{(l)}(n)=0\right) .
$$

(4) Increase in each iteration step: $l=l+1$.

(5) Determination of the value of Engset traffic $A_{j}^{(l)}(n)$ of class $j$ and Pascal traffic $A_{k}^{(l)}(n)$ of class $k$ on the basis of (2.20) and (2.21):

$$
\begin{aligned}
& A_{j}^{(l)}(n)=\alpha_{j}\left[N_{j}-\left(r_{j}^{(l-1)}(n)\right)\right], \\
& A_{k}^{(l)}(n)=\beta_{k}\left[S_{k}+\left(r_{k}^{(l-1)}(n)\right)\right] .
\end{aligned}
$$

(6) Determination of the state probabilities $P^{(l)}(n)$ on the basis of (2.22):

$$
\begin{aligned}
n P^{(l)}(n)= & \sum_{i=1}^{m_{I}} A_{i} t_{i} P^{(l)}\left(n-t_{i}\right)+\sum_{j=1}^{m_{J}} A_{j}^{(l)}\left(n-t_{j}\right) t_{j} P^{(l)}\left(n-t_{j}\right) \\
& +\sum_{k=1}^{m_{K}} A_{k}^{(l)}\left(n-t_{k}\right) t_{k} P^{(l)}\left(n-t_{k}\right) .
\end{aligned}
$$

(7) Determination of the average number of serviced calls $r_{j}^{(l)}(n)$ and $r_{k}^{(l)}(n)$ on the basis of (3.5):

$$
r_{c}^{(l)}(n)= \begin{cases}A_{c}^{(l)}\left(n-t_{c}\right) \frac{P^{(l)}\left(n-t_{c}\right)}{P^{(l)}(n)} & \text { for } 0 \leq n \leq V \\ 0 & \text { otherwise }\end{cases}
$$

(8) Repetition of steps (3)-(6) until predefined accuracy $\epsilon$ of the iterative process is achieved:

$$
\forall_{0 \leq n \leq V} \quad\left(\left|\frac{r_{j}^{(l-1)}(n)-r_{j}^{(l)}(n)}{r_{j}^{(l)}(n)}\right| \leq \epsilon,\left|\frac{r_{k}^{(l-1)}(n)-r_{k}^{(l)}(n)}{r_{k}^{(l)}(n)}\right| \leq \epsilon\right) .
$$


(9) Determination of the blocking probability $E_{c}$ for calls of class $c$ and the loss probability $B_{i}$ for Erlang calls of class $i, B_{j}$ for Engset calls of class $j$, and $B_{k}$ for Pascal calls of class $k$,

$$
\begin{aligned}
E_{c} & =\sum_{n=V-t_{c}+1}^{V} P^{(l)}(n), \\
B_{i} & =E_{i} \\
B_{j} & =\frac{\sum_{n=V-t_{j}+1}^{V} P^{(l)}(n)\left[N_{j}-r_{j}^{(l)}(n)\right] \Lambda_{j}}{\sum_{n=0}^{V} P^{(l)}(n)\left[N_{j}-r_{j}^{(l)}(n)\right] \Lambda_{j}}, \\
B_{k} & =\frac{\sum_{n=V-t_{k}+1}^{V} P^{(l)}(n)\left[S_{k}+r_{k}^{(l)}(n)\right] \gamma_{k}}{\sum_{n=0}^{V} P(n)\left[S_{k}+r_{k}^{(l)}(n)\right] \gamma_{k}} .
\end{aligned}
$$

\subsection{Convergence of the Iterative Process of Estimation of the Average Number of Serviced Engset Calls}

In this section we prove that the process for a determination of the average number of serviced traffic sources proposed in the MIM-BPP method is, in the case of multiservice Engset sources, a convergent process. Thus, the following theorem needs to be proved.

Theorem 3.2. The sequence $\left(r_{j}^{(l)}(n)\right)_{l=0}^{\infty}$ of the average number of serviced class $j$ Engset calls in the system with BPP traffic, where

$$
\begin{gathered}
r_{j}^{(l)}(n)=\frac{\left[N_{j}-r_{j}^{(l-1)}\left(n-t_{j}\right)\right] \alpha_{j} P^{(l-1)}\left(n-t_{j}\right)}{P^{(l-1)}(n)}, \\
r_{j}^{(0)}(n)=0 \quad \text { for } \forall_{1 \leq j \leq m_{J}}, \forall_{0 \leq n \leq V},
\end{gathered}
$$

is convergent.

Proof. In order to prove Theorem 3.2, we are going to show first that each succeeding element of sequence (3.12), starting from the first one, could be represented by finite series:

$$
r_{j}^{(l)}(n)=\sum_{s=1}^{l}(-1)^{s+1} N_{j} \alpha_{j}^{s} \prod_{i=1}^{s} \frac{P^{(l-i)}\left(n-i t_{j}\right)}{P^{(l-i)}\left(n-(i-1) t_{j}\right)} .
$$

Since $r_{j}^{(0)}\left(n-t_{j}\right)=0$, then on the basis of (3.12) for $l=1$

$$
r_{j}^{(1)}(n)=N_{j} \alpha_{j} \frac{P^{(0)}\left(n-t_{j}\right)}{P^{(0)}(n)}
$$


Now, using (3.15), we can determine the value $r_{j}^{(2)}(n)$ for $l=2$ on the basis of (3.12):

$$
r_{j}^{(2)}(n)=\frac{\left[N_{j}-N_{j} \alpha_{j} P^{(0)}\left(n-2 t_{j}\right) / P^{(0)}\left(n-t_{j}\right)\right] \alpha_{j} P^{(1)}\left(n-t_{j}\right)}{P^{(1)}(n)} .
$$

Rearranging (3.16), we can present it in the following way:

$$
r_{j}^{(2)}(n)=N_{j} \alpha_{j} \frac{P^{(1)}\left(n-t_{j}\right)}{P^{(1)}(n)}-N_{j} \alpha_{j}^{2} \frac{P^{(0)}\left(n-2 t_{j}\right) P^{(1)}\left(n-t_{j}\right)}{P^{(0)}\left(n-t_{j}\right) P^{(1)}(n)} .
$$

Proceeding in an analogical way for $l=3$, we obtain

$$
\begin{aligned}
r_{j}^{(3)}(n)= & N_{j} \alpha_{j} \frac{P^{(2)}\left(n-t_{j}\right)}{P^{(2)}(n)} \\
& -N_{j} \alpha_{j}^{2} \frac{P^{(1)}\left(n-2 t_{j}\right) P^{(2)}\left(n-t_{j}\right)}{P^{(1)}\left(n-t_{j}\right) P^{(2)}(n)} \\
& +N_{j} \alpha_{j}^{3} \frac{P^{(0)}\left(n-3 t_{j}\right) P^{(1)}\left(n-2 t_{j}\right) P^{(2)}\left(n-t_{j}\right)}{P^{(0)}\left(n-2 t_{j}\right) P^{(1)}\left(n-t_{j}\right) P^{(2)}(n)} .
\end{aligned}
$$

Generalizing, the value of succeeding element of sequence $\left(r_{j}^{(l)}(n)\right)$ in step $l$ can be expressed by (3.14). Now, setting the limit to infinity $(l \rightarrow \infty)$, we have

$$
\lim _{l \rightarrow \infty} r_{j}^{(l)}(n)=\sum_{s=1}^{\infty}(-1)^{s+1} N_{j} \alpha_{j}^{s} \prod_{i=1}^{s} \frac{P^{(l-i)}\left(n-i t_{j}\right)}{P^{(l-i)}\left(n-(i-1) t_{j}\right)} .
$$

Regardless of the iteration step, for every $n<0$, the probability that system is in a state $n$ is equal to 0 (i.e., $P^{(l)}(n)=0$ ). Thus, we can rewrite (3.19) in the following way:

$$
\lim _{l \rightarrow \infty} r_{j}^{(l)}(n)=\sum_{s=1}^{\left\lfloor n / t_{j}\right\rfloor}(-1)^{s+1} N_{j} \alpha_{j}^{s} \prod_{i=1}^{s} \frac{P^{(l-i)}\left(n-i t_{j}\right)}{P^{(l-i)}\left(n-(i-1) t_{j}\right)}
$$

A series appearing on the right side of (3.20) is finite; therefore, there exists a finite limit of sequence $\left(r_{j}^{(l)}(n)\right)_{l=0}^{\infty}$, which was to be proved.

\subsection{Convergence of the Iterative Process of Estimation of the Average Number of Serviced Pascal Calls}

Let us demonstrate now that the process of a determination of the average number of serviced traffic sources proposed in the MIM-BPP method is a convergent process also in the case of multiservice Pascal sources. The following theorem will be then proved. 
Theorem 3.3. The sequence $\left(r_{k}^{(l)}(n)\right)_{l=0}^{\infty}$ of the average number of serviced class $k$ Pascal sources in the system with BPP traffic, where

$$
\begin{gathered}
r_{k}^{(l)}(n)=\frac{\left[S_{k}+r_{k}^{(l-1)}\left(n-t_{k}\right)\right] \beta_{k} P^{(l-1)}\left(n-t_{k}\right)}{P^{(l-1)}(n)}, \\
r_{k}^{(0)}(n)=0 \quad \text { for } \forall_{1 \leq k \leq m_{J}}, \forall_{0 \leq n \leq V},
\end{gathered}
$$

is convergent.

Proof. Proceeding in the analogical way as we did in the case of sequence (3.12), we can prove that the elements of sequence $\left(r_{k}^{(l)}(n)\right)_{l=0}^{\infty}$ can be expressed by the following expression:

$$
r_{k}^{(l)}(n)=\sum_{j=1}^{l} S_{k} \beta_{k}^{j} \prod_{i=1}^{j} \frac{P^{(l-i)}\left(n-i t_{k}\right)}{P^{(l-i)}\left(n-(i-1) t_{k}\right)} .
$$

Therefore, in order to show that sequence $\left(r_{k}^{(l)}(n)\right)_{l=0}^{\infty}$ is convergent, we only need to prove that for $l \rightarrow \infty$ the series

$$
\sum_{j=1}^{l} S_{k} \beta_{k}^{j} \prod_{i=1}^{j} \frac{P^{(l-i)}\left(n-i t_{k}\right)}{P^{(l-i)}\left(n-(i-1) t_{k}\right)}
$$

is convergent.

Consider the elements of series (3.24):

$$
b_{j}=S_{k} \beta_{k}^{j} \prod_{i=1}^{j} \frac{P^{(l-i)}\left(n-i t_{k}\right)}{P^{(l-i)}\left(n-(i-1) t_{k}\right)} .
$$

The elements of series $\left(b_{j}\right)_{j=1}^{\infty}$ are positive, which means that we can use the ratio test (d'Alembert criterium) for convergence to prove that series is convergent (if in series $\sum_{n=1}^{\infty} u_{n}$ with positive terms beginning from certain place $N$ (this means for all $n \geq N$ ), then the ratio of arbitrary term $u_{n+1}$ to previous term $u_{n}$ is permanently less than number $p$ less than 1; i.e, if $u_{n+1} / u_{n} \leq p<1$ for all $n \geq N$, then series $\sum_{n=1}^{\infty} u_{n}$ is convergent [33]). The ratio of two consecutive elements of sequence $\left(b_{j}\right)_{j=1}^{\infty}$ is equal to

$$
\frac{b_{j+1}}{b_{j}}=\beta_{k} \frac{P^{(l-(j+1))}\left(n-(j+1) t_{k}\right)}{P^{(l-(j+1))}\left(n-j t_{k}\right)} .
$$

For $j \rightarrow \infty$ numerator and denominator of (3.26) converge to 0 . Note also that the numerator converges to 0 faster than the denominator. Hence, $\lim _{j \rightarrow \infty}\left(b_{j+1} / b_{j}\right)$ is equal to 0 , that is, is permanently less than 1 . Therefore, by virtue of the ratio test (d'Alembert criterium) for convergence series (3.24) is convergent. Thus, sequence (3.21) is convergent as well. 


\subsection{Advantages and Possible Applications of MIM-BPP Method}

The presented iterative algorithm for systems with state-independent admission process (i.e., the full-availability group) makes it possible to determine exactly the occupancy distribution and the blocking and loss probabilities in systems that service Erlang (Poisson distribution of call streams), Engset (binomial distribution of call streams), and Pascal traffic streams (negative binomial distribution of call stream). The call stream of the types investigated in the paper are typical streams to be considered in traffic theory. They are used for modelling at the call level, where any occupancy of resources of the system, for example, effected by a telephone conversation or by a packet stream with characteristics defined at the packet level, can be treated as a call [11]. In the case of the Integrated Services Digital Networks, resource occupancies were in the main related to voice transmission, whereas nowadays a call is understood to be a packet stream to which appropriate equivalent bandwidth is assigned [3436], and then the demanded resources, as well as the capacity of the system, are discretized [7]. In the case of wired systems, the most important is the Poisson stream and the consequent Erlang traffic stream. This stream assumes stable intensity of generating calls, independent of the number of calls that are already being serviced. In the case of wireless systems, it was soon noticed that, because of the limited number of subscribers serviced within a given area, the application of the Erlang model for certain traffic classes could lead to erroneous estimation of the occupancy distribution. Hence, for certain traffic classes, the application of the Engset model was proposed, initially for single-service (single-rate) systems and then for multiservice (multirate) systems [3,4]. In general, the Engset distribution is used to model systems with noticeable limitation of the number of users. Currently, the main practical scope for the usage of the Pascal distribution is a simplified modelling of systems with overflow traffic [11]. The presented algorithm makes it then possible to determine traffic characteristics for all three call (traffic) streams considered in traffic theory.

The application of the notion of the basic bandwidth unit (BBU) used in the notation of the presented method makes it possible to obtain high universality for the method. BBU is determined as the highest common divisor of all demands that are offered to the system. Depending on a system under consideration, the basic bandwidth unit can be expressed in bits per second or as the percentage of the occupancy of the radio interface (the so-called interference load) [4, 37]. In the presented method for modelling multirate systems with BPP traffic streams, both required resources and the capacity of the system are expressed as the multiplicity of BBU. The method can be thus applied to model both wired broadband integrated services networks as well as wireless networks (UMTS/WCDMA networks in particular).

The algorithm worked out for modelling systems with BPP traffic can be treated as an extension to the Kaufman-Roberts model $[24,25]$ that has been worked out for systems with Poisson traffic streams only. Both the algorithm proposed by Kaufman-Roberts and the algorithm presented in the paper are exact algorithms. Having an exact formula as a base, the algorithm can be extended-analogously as in the case of the Kaufman-Roberts formula for systems with Erlang traffic-into systems with state-dependent call admission process and BPP traffic. In the case of communication system, state dependence in the call admission process results mainly from the introduction of the control policy in allocating resources for calls of individual traffic classes (reservation mechanism [32], threshold mechanism [38]) or a particular structure of the system (e.g., a limited-availability group [5]). An extension of the scope in which the presented algorithm can be applied, including systems with statedependent call admission process, entails only the introduction of the additional transition 
Table 1: Relative errors of the number of busy class 3 sources in relation to the number of iterations.

\begin{tabular}{lcccc}
\hline$a$ & \multicolumn{2}{c}{ The step of iteration } & 7 \\
\hline 0.3 & 2 & 3 & 5 & $1.11 E-10$ \\
0.4 & $3.17 E-02$ & $7.17 E-04$ & $2.97 E-07$ & $7.68 E-11$ \\
0.5 & $2.89 E-02$ & $3.51 E-04$ & $1.65 E-07$ & $8.06 E-11$ \\
0.6 & $3.43 E-02$ & $4.86 E-04$ & $1.18 E-07$ & $1.09 E-10$ \\
0.7 & $3.97 E-02$ & $6.36 E-04$ & $1.93 E-07$ & $1.42 E-10$ \\
0.8 & $4.52 E-02$ & $8.02 E-04$ & $2.95 E-07$ & $2.37 E-10$ \\
0.9 & $5.06 E-02$ & $9.87 E-04$ & $4.29 E-07$ & $3.75 E-10$ \\
1.0 & $5.59 E-02$ & $1.19 E-03$ & $5.98 E-07$ & $5.68 E-10$ \\
1.1 & $6.11 E-02$ & $1.41 E-03$ & $8.14 E-07$ & $8.33 E-10$ \\
1.2 & $6.62 E-02$ & $1.64 E-03$ & $1.08 E-06$ & $1.20 E-09$ \\
\hline
\end{tabular}

coefficient [32], without further changes, depending on the considered system. It should be stressed that such a universality cannot be achieved by the convolution algorithm also worked out for systems with state-independent call admission process only.

\subsection{Numerical Examples}

The paper introduces a formula that makes it possible to determine exactly the occupancy distribution in systems with state-independent call admission process. It is then demonstrated that the algorithm for a determination of the average number of serviced traffic sources of particular classes used in the MIM-BPP method is convergent.

In order to present the convergence of the MIM-BPP method (the number of required iterations), in Table 1 the results of relative errors of the number of busy class 3 sources in the full-availability group with the capacity equal to $80 \mathrm{BBUs}$ are contained (with the instance of calls of class 1 and 2, the number of required iterations is lower than in the case of the presented results for class 3$)$. The results are presented depending on the average value of traffic offered to a single bandwidth unit of the group: $a=\left(\sum_{i=1}^{m_{I}} A_{i} t_{i}+\sum_{j=1}^{m_{J}} N_{j} \alpha_{j} t_{j}+\right.$ $\left.\sum_{k=1}^{m_{K}} S_{k} \beta_{k} t_{k}\right) / V$. The group was offered three traffic classes, that is, Erlang traffic class: $t_{1}=$ $1 \mathrm{BBU}$, Engset traffic class: $t_{2}=4 \mathrm{BBUs}, N_{2}=60$, and Pascal traffic class: $t_{3}=10 \mathrm{BBUs}, S_{3}=80$. The results presented in Table 1 indicate that the proposed iterative method converges very quickly.

In this section we limit ourselves to just presenting the results of the convergence of the presented algorithm for one selected system. A comparison of the analytical results for the blocking/loss probability with the results of the simulation is presented in earlier works, for example, $[4,32]$, in which it was still assumed that the presented analytical method was an approximate method.

\section{Conclusion}

In the paper recurrent equations describing—at the macrostate level一the service process in the full-availability group with multirate BPP traffic were derived. The derived equations made it possible to formulate an exact iterative algorithm for determining the occupancy 
distribution, blocking probability, and loss probability of calls of particular classes offered to the system. The convergence of the proposed process of estimating the average number of busy sources of Engset and Pascal traffic was proved.

\section{References}

[1] D. Staehle and A. Mäder, "An analytic approximation of the uplink capacity in a UMTS network with heterogeneous traffic," in Proceedings of the 18th International Teletraffic Congress (ITC '03), pp. 81-91, Berlin, Germany, 2003.

[2] M. Głąbowski, M. Stasiak, A. Wiśniewski, and P. Zwierzykowski, "Blocking probability calculation for cellular systems with WCDMA radio interface servicing PCT1 and PCT2 multirate traffic," IEICE Transactions on Communications, vol. E92-B, no. 4, pp. 1156-1165, 2009.

[3] G. A. Kallos, V. G. Vassilakis, and M. D. Logothetis, "Call-level performance analysis of a WCDMA cell with finite population and interference cancellation," The European Transactions on Telecommunications, vol. 22, no. 1, pp. 25-30, 2011.

[4] M. Stasiak, M. Głąbowski, A. Wiśniewski, and P. Zwierzykowski, Modeling and Dimensioning of Mobile Networks, Wiley, 2011.

[5] M. Głąbowski, A. Kaliszan, and M. Stasiak, "Modeling product-form state-dependent systems with BPP traffic," Performance Evaluation, vol. 67, no. 3, pp. 174-197, 2010.

[6] F. P. Kelly, “Loss networks," The Annals of Applied Probability, vol. 1, no. 3, pp. 319-378, 1991.

[7] J. W. Roberts, V. Mocci, and I. Virtamo, Broadband Network Teletraffic, Final Report of Action COST 242, Commission of the European Communities, Springer, Berlin, Germany, 1996.

[8] M. Pióro, J. Lubacz, and U. Körner, "Traffic engineering problems in multiservice circuit switched networks," Computer Networks and ISDN Systems, vol. 20, pp. 127-136, 1990.

[9] L. A. Gimpelson, "Analysis of mixtures of wide and narrow-band traffic," IEEE Transactions on Communication Technology, vol. 13, no. 3, pp. 258-266, 1953.

[10] J. M. Aein, "A multi-user-class, blocked-calls-cleared, demand access model," IEEE Transactions on Communications, vol. 26, no. 3, pp. 378-384, 1978.

[11] V. B. Iversen, Teletraffic Engineering Handbook, ITU-D, Study Group 2, Question 16/2, Geneva, Switzerland, 2005.

[12] K. W. Ross, Multiservice Loss Models for Broadband Telecommunication Network, Springer, London, UK, 1995.

[13] G. M. Stamatelos and J. F. Hayes, “Admission-control techniques with application to broadband networks," Computer Communications, vol. 17, no. 9, pp. 663-673, 1994.

[14] J. Conradt and A. Buchheister, "Considerations on loss probability of multi-slot connections," in Proceedings of the 11th International Teletraffic Congress (ITC'85), pp. 4.4B-2.1, Kyoto, Japan, 1985.

[15] V. B. Iversen, "The exact evaluation of multi-service loss systems with access control," in Proceedings of the 7th Nordic Teletraffic Seminar (NTS '87), pp. 56-61, Lund, Sweden, August 1987.

[16] M. E. Beshai and D. R. Manfield, "Multichannel services performance of switching networks," in Proceedings of the 12th International Teletraffic Congress (ITC '88), pp. 857-864, Elsevier, Torino, Italy, 1988.

[17] M. Stasiak, "An approximate model of a switching network carrying mixture of different multichannel traffic streams," IEEE Transactions on Communications, vol. 41, no. 6, pp. 836-840, 1993.

[18] L. E. N. Delbrouck, "On the steady-state distribution in a service facility carrying mixtures of traffic with different peakedness factors and capacity requirements," IEEE Transactions on Communications, vol. 31, no. 11, pp. 1209-1211, 1983.

[19] J. S. Kaufman, "Blocking with retrials in a completely shared resource environment," Performance Evaluation, vol. 15, no. 2, pp. 99-113, 1992.

[20] H. L. Hartmann and M. Knoke, "The one-level functional equation of multi-rate loss systems," The European Transactions on Telecommunications, vol. 14, no. 2, pp. 107-118, 2003.

[21] V. G. Vassilakis, I. D. Moscholios, and M. D. Logothetis, "Call-level performance modelling of elastic and adaptive service-classes with finite population," IEICE Transactions on Communications, vol. E91-B, no. 1, pp. 151-163, 2008.

[22] M. Głąbowski, A. Kaliszan, and M. Stasiak, "Asymmetric convolution algorithm for blocking probability calculation in full-availability group with bandwidth reservation," IET Circuits, Devices and Systems, vol. 2, no. 1, pp. 87-94, 2007. 
[23] M. Głąbowski, A. Kaliszan, and M. Stasiak, "On the application of the asymmetric convolution algorithm in modeling of full-availability group with bandwidth reservation," in Proceedings of the 20th International Teletraffic Congress (ITC '07), Managing Traffic Performance in Converged Networks, L. Mason, T. Drwiega, and J. Yan, Eds., vol. 4516 of Lecture Notes in Computer Science, pp. 878-889, Springer, Ottawa, Canada, June 2007.

[24] J. S. Kaufman, "Blocking in a shared resource environment," IEEE Transactions on Communications, vol. 29, no. 10, pp. 1474-1481, 1981.

[25] J. W. Roberts, "A service system with heterogeneous user requirements-application to multiservice telecommunications systems," in Proceedings of the International Conference on Performance of Data Communication Systems and Their Applications, G. Pujolle, Ed., pp. 423-431, North Holland, Amsterdam, The Netherlands, 1981.

[26] J. P. Labourdette and G. W. Hart, "Blocking probabilities in multitraffic loss systems: insensitivity, asymptotic behavior and approximations," IEEE Transactions on Communications, vol. 40, no. 8, pp. 1355-1367, 1992.

[27] D. Tsang and K. W. Ross, "Algorithms to determine exact blocking probabilities for multirate tree networks," IEEE Transactions on Communications, vol. 38, no. 8, pp. 1266-1271, 1990.

[28] Z. Dziong and J. W. Roberts, "Congestion probabilities in a circuit-switched integrated services network," Performance Evaluation, vol. 7, no. 4, pp. 267-284, 1987.

[29] E. Pinsky and A. E. Conway, "Computational algorithms for blocking probabilities in circuit-switched networks," Annals of Operations Research, vol. 35, no. 1, pp. 31-41, 1992.

[30] G. A. Awater and H. A. B. van de Vlag, "Exact computation of time and call blocking probabilities in large, multi-traffic, multi-resource loss systems," Performance Evaluation, vol. 25, no. 1, pp. 41-58, 1996.

[31] M. Głąbowski and M. Stasiak, "An approximate model of the full-availability group with multi-rate traffic and a finite source population," in Proceedings of the 3rd Polish-German Teletraffic Symposium (PGTS '04), P. Buchholtz, R. Lehnert, and M. Pióro, Eds., pp. 195-204, VDE Verlag, Dresden, Germany, September 2004.

[32] M. Głąbowski, "Modelling of state-dependent multirate systems carrying BPP traffic," Annals of Telecommunications, vol. 63, no. 7-8, pp. 393-407, 2008.

[33] W. Rudin, Principles of Mathematical Analysis (International Series in Pure E Applied Mathematics), McGraw-Hill Science/Engineering/Math, New York, NY, USA, 1976.

[34] F. P. Kelly, “Notes on effective bandwidth," Tech. Rep., University of Cambridge, 1996.

[35] L. Aspirot, P. Belzarena, P. Bermolen, A. Ferragut, G. Perera, and M. Simon, "Quality of service parameters and link operating point estimation based on effective bandwidths," Performance Evaluation, vol. 59, no. 2-3, pp. 103-120, 2005.

[36] S. Bodamer and J. Charzinski, "Evaluation of effective bandwidth schemes for self-similar traffic," in Proceedings of the 13th ITC Specialist Seminar on IP Traffic Measurement, Modeling and Management, pp. 21.1-21.10, Monterey, Calif, USA, 2000.

[37] H. Holma and A. Toskala, WCDMA for UMTS: HSPA Evolution and LTE, John Wiley \& Sons, New York, NY, USA, 2007.

[38] M. Głąbowski, “Continuous threshold model for multi-service wireless systems with PCT1 and PCT2 traffic," in Proceedings of the 7th International Symposium on Communications and Information Technologies (ISCIT '07), pp. 427-432, Sydney, Australia, October 2007. 


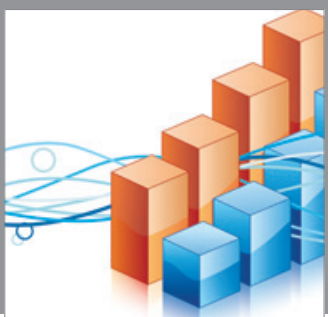

Advances in

Operations Research

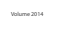

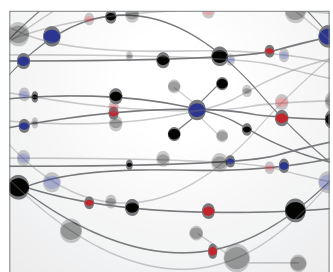

\section{The Scientific} World Journal
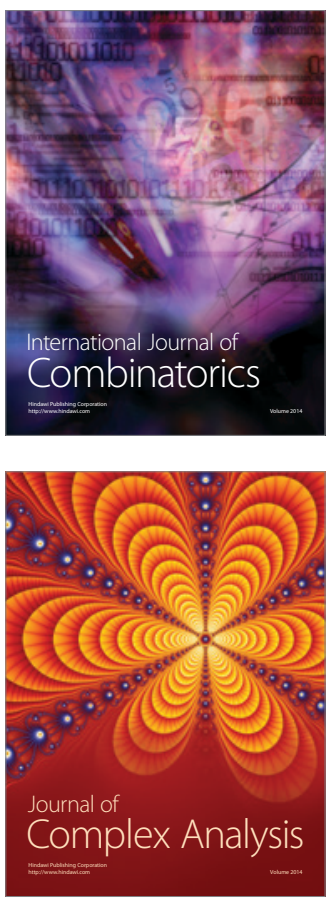

International Journal of

Mathematics and

Mathematical

Sciences
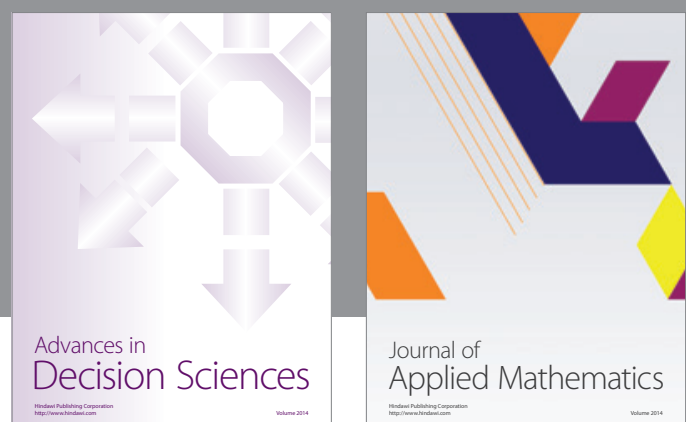

Journal of

Applied Mathematics
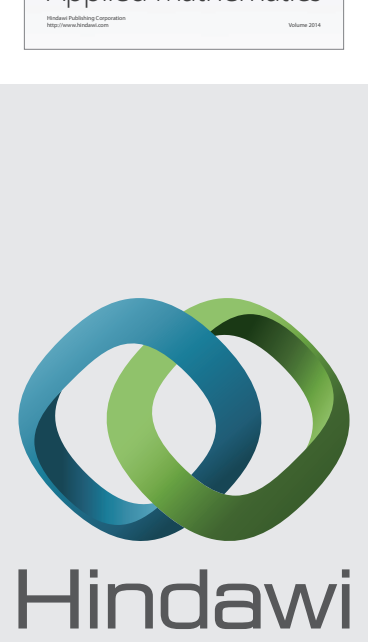

Submit your manuscripts at http://www.hindawi.com
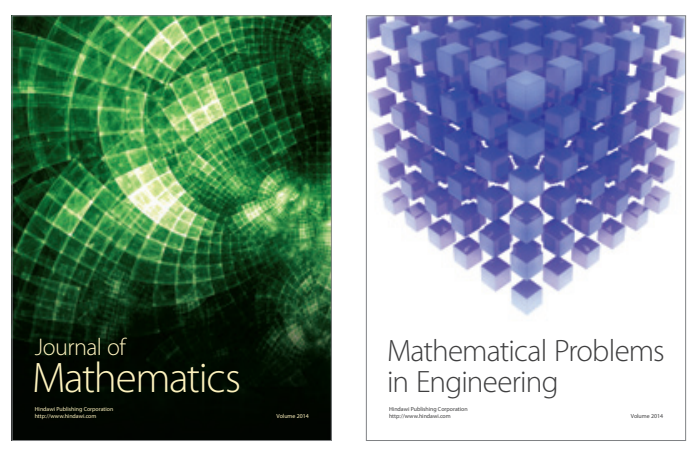

Mathematical Problems in Engineering
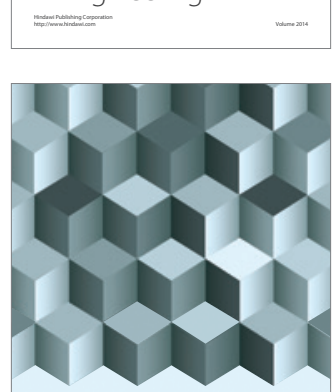

Journal of

Function Spaces
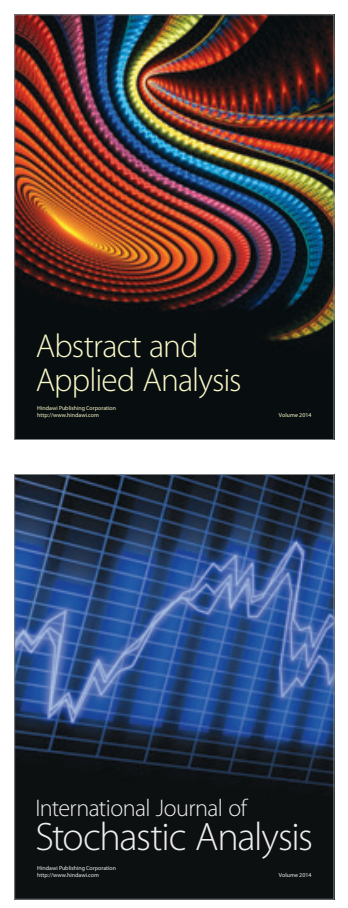

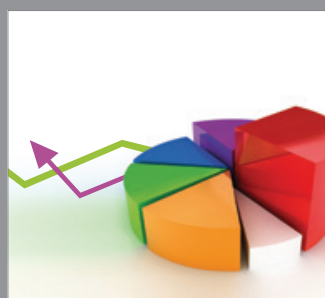

ournal of

Probability and Statistics

Promensencen
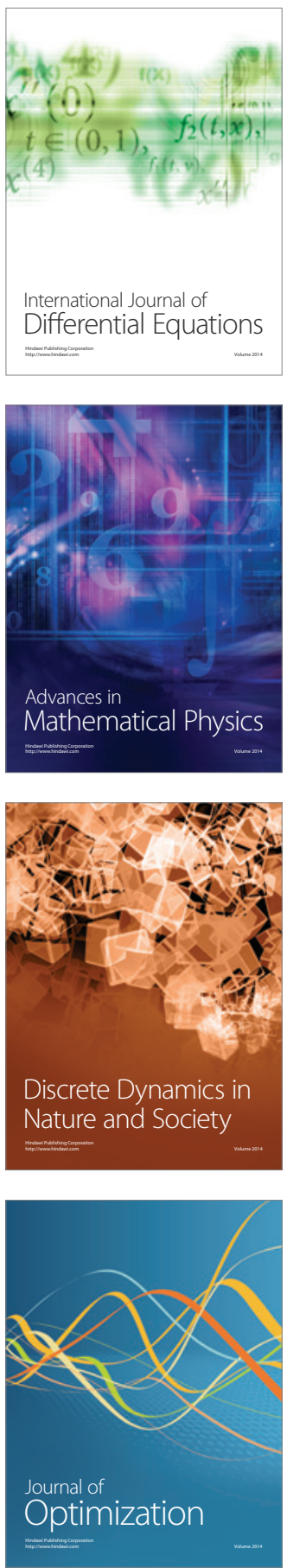